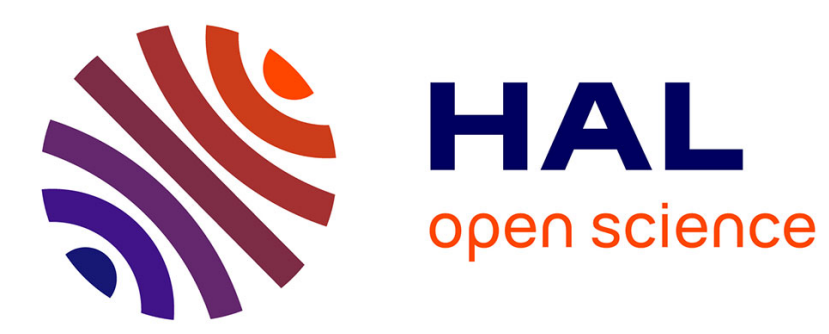

\title{
Capacité de diffusion pulmonaire avant un triathlon et 24 heures après la compétition
}

\author{
Alain Boussana, Olivier Hue, Maurice Hayot, Stefan Matecki, Michèle \\ Ramonatxo, Daniel Le Gallais
}

\section{- To cite this version:}

Alain Boussana, Olivier Hue, Maurice Hayot, Stefan Matecki, Michèle Ramonatxo, et al.. Capacité de diffusion pulmonaire avant un triathlon et 24 heures après la compétition. Science \& Sports, 2000, 15 (5), pp.245-247. 10.1016/S0765-1597(00)80035-0 . hal-00720522

\section{HAL Id: hal-00720522 https://hal.univ-antilles.fr/hal-00720522}

Submitted on 24 Jul 2012

HAL is a multi-disciplinary open access archive for the deposit and dissemination of scientific research documents, whether they are published or not. The documents may come from teaching and research institutions in France or abroad, or from public or private research centers.
L'archive ouverte pluridisciplinaire HAL, est destinée au dépôt et à la diffusion de documents scientifiques de niveau recherche, publiés ou non, émanant des établissements d'enseignement et de recherche français ou étrangers, des laboratoires publics ou privés. 


\title{
Capacité de diffusion pulmonaire avant un triathlon et 24 heures après la compétition
}

\author{
A. Boussana', O. Hue ${ }^{1}$, M. Hayot ${ }^{2}$, S. Matécki², M. Ramonatxo², D. Le Gallais ${ }^{1}$ \\ 'Laboratoire sport performance santé, faculté des sciences du sport, 700, avenue du Pic-Saint-Loup, 34090 Montpellier : \\ -laboratoire de physiologie des interactions, centre hospitalier universitaire Arnaud-de-Villeneure. 34295 Montpellier
cedex 5. France
}

\begin{abstract}
Résumé
Objectifs. - Évaluer les effets du triathlon classique $(1,5 \mathrm{~km}$ natation, $40 \mathrm{~km}$ cyclisme, $10 \mathrm{~km}$ course à pied) sur la capacité de diffusion pulmonaire $\left(\mathrm{DL}_{\mathrm{CO}}\right) 24$ heures après la compétition.

Méthodes et résultats. - Dix triathlètes masculins $(21,5 \pm 2,8$ ans) ont effectué une spirométrie et une mesure de la capacité de diffusion pulmonaire avant la compétition (pré-T) et 24 heures après l'épreuve (postT). Les résultats montrent une diminution significative du volume expiratoire maximal en $1 \mathrm{~s}\left(5,17 \pm 0,4 \mathrm{L.s}^{-1}\right.$ contre 4,09 $\left.\pm 0,6 \mathrm{~L} . \mathrm{s}^{-1}\right)$ et du débit expiratoire maximal $25-75 \%$ de la capacité vitale $\left(6,98 \pm 0,2 \mathrm{~L} . \mathrm{s}^{-1}\right.$ contre $\left.4,90 \pm 0,2 \mathrm{~L} . \mathrm{s}^{-1}\right)$. En revanche, les résultats ne montrent pas de différence significative entre les $\mathrm{DL}_{\mathrm{Co}}$ pré-T et post-T $\left(38,1 \pm 3,3 \mathrm{~mL} \cdot \mathrm{min}^{-1} \cdot \mathrm{mmHg}^{-1}\right.$ contre $\left.37,1 \pm 3,7 \mathrm{~mL} \cdot \mathrm{min}^{-1} \cdot \mathrm{mmHg}^{-1}\right)$ ni les ratios $\mathrm{DL}_{\mathrm{CO}} \mathrm{NA}\left(5,59 \pm 0,5 \mathrm{~mL} \cdot \mathrm{min}^{-1} \cdot \mathrm{mmHg}^{-1} \cdot \mathrm{A}^{-1}\right.$ contre $\left.5,55 \pm 0,7 \mathrm{~mL} \cdot \mathrm{min}^{-1} \cdot \mathrm{mmHg}^{-1} \cdot \mathrm{A}^{-1}\right)$.

Conclusion. - La DL $L_{C O}$ est normale chez les triathlètes 24 heures après une compétition classique. Ceci peut traduire l'absence de lésions alvéolocapillaires pendant le triathlon. La baisse de la $D L_{c o}$ rapportée deux heures après un exercice dans la littérature peut s'expliquer par la baisse du volume sanguin pulmonaire qui apparaît comme un phénomène transitoire. Cependant, la baisse des débits pulmonaires observée 24 heures après le triathlon suggère la persistance chez les triathlètes d'un syndrome obstructif bronchique. (C) 2000 Éditions scientifiques et médicales Elsevier SAS
\end{abstract}

capacité de diffusion pulmonaire / $\mathrm{DL}_{\mathrm{co}} /$ spirométrie / triathlon

Summary - Pulmonary diffusing capacity before and $24 \mathrm{~h}$ after a triathlon.

Objectives. - Investigate the effects of a classic triathlon $(1.5 \mathrm{~km}$ swim, $40 \mathrm{~km}$ cycle, and $10 \mathrm{~km}$ run) on pulmonary diffusing capacity ( $\left.D L_{c o}\right)$.

Methods and results. - Ten male triathletes aged $21.5 \pm 2.8 \mathrm{yr}$ underwent a spirometry and measurement of pulmonary diffusing capacity $\left(D L_{c o}\right)$ before the competition (pre-T) and $24 \mathrm{hr}$ after (post-T). $D L_{c o}$ and the ratio of $D L_{c o}$ to alveolar volume (VA) $\left(D L_{C O} N A\right)$ were successively calculated. Results showed a significant decrease in forced expiratory volume in $1 \mathrm{~s}$ (FEV $; 5.17 \pm 0.41 . \mathrm{s}^{-1} \mathrm{vs}$ $4.09 \pm 0.6 \mathrm{l}^{-1}$ ) and mean forced expiratory flow during the middle half of vital capacity ( $F E F F_{25-75 \%}$; $6.98 \pm 0.21 .5^{-1}$ versus $\left.4.90 \pm 0.21 .5^{-1}\right)$. In contrast we did not observe significant difference in $D L_{c o}$ and $D L_{c o} V A$ between pre- $T$ and post- $T$ values.

Conclusion. $-D L_{C O}$ was not impaired $24 \mathrm{hr}$ after a classic triathlon. This result could traduce the absence of alveolo-capillary membrane lesion during a competition. The decrease in $D L_{c o}$ reported $2 \mathrm{hr}$ after exercise in the literature may be explained by the decrease in pulmonary blood volume, which appears to be a transient phenomenon. However, the decrease in pulmonary flows suggested the existence of a pulmonary obstructive syndrome. (O 2000 Éditions scientifiques et médicales Elsevier SAS

pulmonary diffusing capacity / $\mathrm{DL}_{\mathrm{co}} /$ spirometry / triathlon 
La capacité de diffusion pulmonaire $\left(\mathrm{DL}_{\mathrm{CO}}\right)$ augmente au cours de l'exercice maximal en laboratoire [1] et diminue pendant la récupération [2]. Il en est de même au cours des épreuves de terrain [3]. Après un exercice d'intensité maximale, Rasmussen et al. [2] ont montré que la normalisation de la $\mathrm{DL}_{\mathrm{CO}}$ était obtenue à la $20^{\mathrm{e}}$ heure de la récupération ; après le même type d'exercice, Hopkins et al. [4] ont montré par l'étude du liquide de lavage broncho-alvéolaire une altération de la membrane alvéolocapillaire. Au contraire, après un exercice d'intensité sousmaximale, correspondant à une heure de cycloergomètre à 75-80\% de la $\dot{V O}_{2} \max$, Hopkins et al. [5] ont montré que la membrane alvéolocapillaire ne présentait pas d'altération. Les principaux mécanismes physiopathologiques évoqués pour expliquer la baisse de $\mathrm{DL}_{\mathrm{CO}}$ statistiquement significative en post-exercice par rapport à l'état basal sont : une altération de la membrane alvéolocapillaire, une diminution du volume sanguin pulmonaire et un œdème pulmonaire et/ou alvéolaire. En outre, notre équipe [3] a observé une baisse de la $\mathrm{DL}_{\mathrm{CO}}$ deux heures après un triathlon et l'étude radiographique pulmonaire réalisée a permis de suggérer la présence d'un œdème pulmonaire interstitiel. Afin d'approcher les mécanismes mis en jeu dans la diminution de la $\mathrm{DL}_{\mathrm{CO}}$ après l'exercice, nous avons comparé la $\mathrm{DL}_{\mathrm{CO}}$ d'athlètes avant un triathlon et 24 heures après la compétition.

\section{MATÉRIEL ET MÉTHODE}

\section{Sujets}

Dix triathlètes masculins âgés de 19 à 26 ans $(21,5 \pm 2,8$ ans) ont participé à cette étude. Les sujets avaient un entraînement hebdomadaire de $14 \pm 3 \mathrm{~km}$ de natation, $50 \pm 14 \mathrm{~km}$ de cyclisme et $44 \pm 13 \mathrm{~km}$ de course à pied. Ils étaient de niveau national et régional.

\section{Spirométrie}

La capacité vitale $(\mathrm{CV})$, la capacité pulmonaire totale (CPT), le volume résiduel (VR), la capacité résiduelle fonctionnelle (CRF), le volume expiratoire maximal en une seconde (VEMS), le rapport de Tiffeneau (VEMS/ CVF) et les débits instantanés à $25-75 \%, 25,50$ et $75 \%$ de la capacité vitale ont été mesurés avec un spiromètre (Pulmonet III ${ }^{\mathrm{TM}}$, Sensordimedics, Bilthovel. Holland).

\section{Mesure de la $\mathrm{DL}_{\mathrm{co}}$}

Cette mesure a été réalisée en apnée par la technique du transfert test Morgan. La $\mathrm{DL}_{\mathrm{CO}}$ est la quantité de monoxyde de carbone $(\mathrm{CO})$ diffusée à travers la membrane alvéolocapillaire en une minute. Le sujet inhale un mélange gazeux, reste ensuite en apnée pendant dix

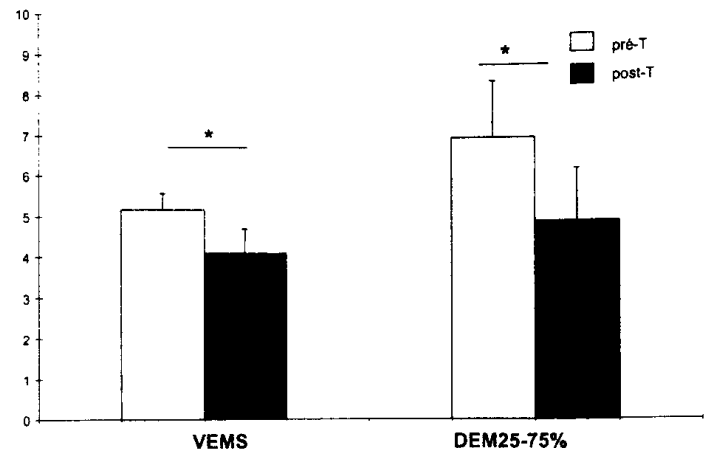

Figure 1. Débits pulmonaires (VEMS, DEM 25-75\%) observés avant (pré-T) et 24 heures après le triathlon (post-T). ${ }^{*}$ différence significative.

secondes puis il effectue une expiration forcée. La $\mathrm{DL}_{\mathrm{CO}}$ et le ratio $\mathrm{DL}_{\mathrm{CO}} / \mathrm{VA}$ sont simultanément mesurés.

\section{Protocole}

Deux à cinq jours avant le triathlon et 24 heures après la compétition $(22,4 \pm 2,6 \mathrm{~h})$, chaque sujet a effectué une épreuve de spirométrie et une mesure de la $\mathrm{DL}_{\mathrm{CO}}$.

\section{Le triathlon}

Il s'agissait du triathlon de la Grande-Motte (34, Montpellier, France), épreuve de distance olympique et donc composée de $1,5 \mathrm{~km}$ de natation, $40 \mathrm{~km}$ de cyclisme et $10 \mathrm{~km}$ de course à pied.

\section{Statistiques}

Le traitement statistique a comporté un test $t$ de student pour des mesures répétées afin de comparer les valeurs avant le triathlon (pré-T) et après le triathlon (post-T). Le seuil de significativité était fixé à $p<0,05$.

\section{RÉSULTATS}

\section{Spirométrie}

Les mesures des volumes pulmonaires du pré-T et du post-T ne montrent pas de différence significative : $\mathrm{CV}$ $(6,05 \pm 0,6 \mathrm{~L}$ contre $6,11 \pm 0,6 \mathrm{~L})$. CPT $(7,47 \pm 0,4 \mathrm{~L}$ contre 7,71 $\pm 0,7 \mathrm{~L})$. VR $(1,42 \pm 0,2 \mathrm{~L}$ contre $1,6 \pm 0,3 \mathrm{~L})$. Par ailleurs nous avons observé une diminution significative des débits pulmonaires entre les mesures pré-T et post-T (figure 1). 

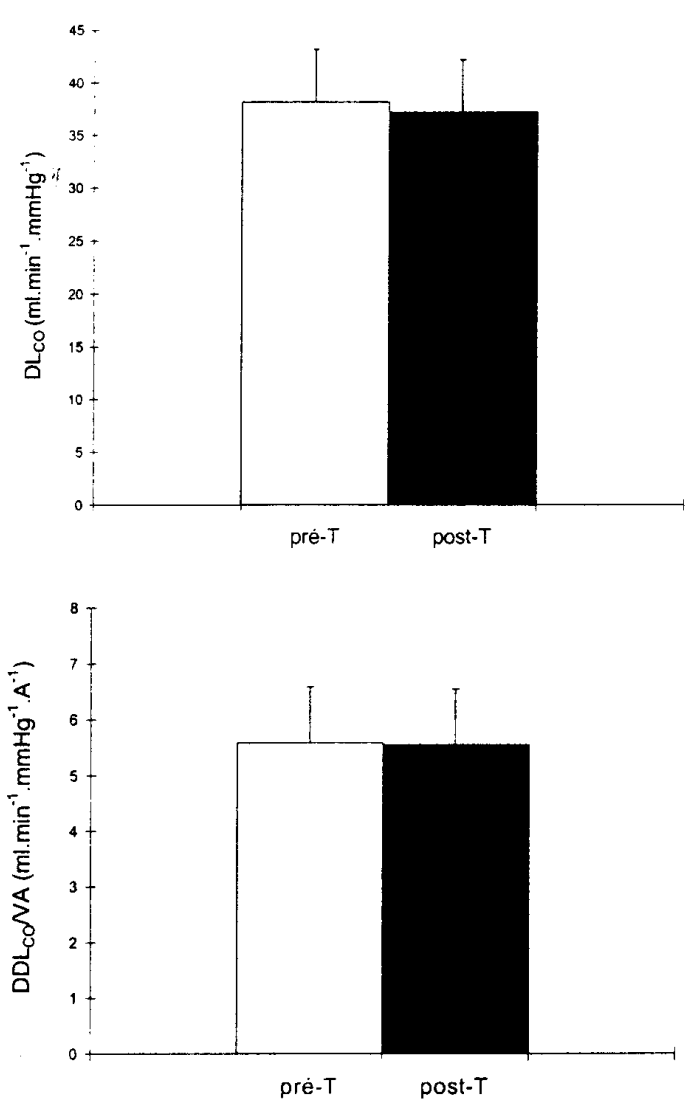

Figure 2. Capacité de diffusion pulmonaire au monoxyde de carbone $\left(\mathrm{DL}_{\mathrm{CO}}\right)$ et ratio $\mathrm{DL}_{\mathrm{CO}}$ sur volume alvéolaire $\left(\mathrm{DL}_{\mathrm{CO}} / \mathrm{VA}\right)$ observés avant (pré-T) et 24 heures après le triathlon (post-T). Absence de différence significative.

\section{$\mathrm{DL}_{\text {Co }}$}

Les valeurs de $\mathrm{DL}_{\mathrm{CO}}$ mesurées en pré-T et post-T n'ont pas montré de différence significative $(38,1 \pm 3,3$ $\mathrm{mL} \cdot \mathrm{min}^{-1} \cdot \mathrm{mmHg}^{-1}$ contre $\left.37,1 \pm 3,7 \mathrm{~mL} \cdot \mathrm{min}^{-1} \cdot \mathrm{mmHg}^{-1}\right)$. Il en a été de même des valeurs du ratio $\mathrm{DL}_{\mathrm{CO}} / \mathrm{VA}(5,59 \pm$ $0,5 \mathrm{~mL} \cdot \mathrm{min}^{-1} \cdot \mathrm{mmHg}^{-1} \cdot \mathrm{A}^{-1}$ contre $5,55 \pm 0,7 \mathrm{~mL} \cdot \mathrm{min}^{-1}$. $\mathrm{mmHg}-1 . \mathrm{A}^{-1}$ ) (figure 2).

\section{DISCUSSION}

La présente étude montre que la $\mathrm{DL}_{\mathrm{CO}}$ des triathlètes est normale 24 heures après une compétition classique. Après un exercice de longue durée et d'intensité sous-maximale comme un triathlon et en l'absence supposée d'altération de la membrane alvéolocapillaire [5], les deux principaux mécanismes physiopathologiques susceptibles d'expliquer la baisse de la $\mathrm{DL}_{\mathrm{CO}}$ sont la diminution du volume sanguin pulmonaire et l'œdème pulmonaire interstitiel et/ ou alvéolaire [2]. Ce dernier mécanisme, dû à un trouble de la perméabilité membranaire au cours de l'exercice, est en relation avec des phénomènes inflammatoires, ainsi qu'avec l'élévation de la pression capillaire pulmonaire. On peut émettre l'hypothèse que la baisse du volume sanguin pulmonaire rapportée après l'exercice, indépendamment de la nature et de l'intensité de celui-ci [6] s'accompagne d'une baisse de la pression capillaire pulmonaire, facteur déterminant pour la résorption de l'œdème interstitiel ou alvéolaire contracté pendant l'exercice. Ainsi, les variations de la $\mathrm{DL}_{\mathrm{CO}}$ observées au cours du triathlon et pendant la récupération paraissent donc dépendre principalement des mécanismes hémodynamiques localisés au niveau des capillaires pulmonaires, mécanismes transitoires et rapidement réversibles. Cependant, la baisse significative des débits pulmonaires 24 heures après le triathlon suggère la persistance d'un adème péribronchoalvéolaire, traduisant la résorption incomplète d'un œdème pulmonaire ou interstitiel chez les sujets.

\section{CONCLUSION}

En conclusion, notre étude montre que la $\mathrm{DL}_{\mathrm{CO}}$ des triathlètes est normale 24 heures après une compétition classique. Les mécanismes physiopathologiques mis en jeu sont : le débit sanguin pulmonaire et l'état de la membrane alvéolocapillaire, qui paraissent être l'objet de modifications peu graves, transitoires, et réversibles. La diminution des débits pulmonaires suggère la persistance d'un œdème péribronchoalvéolaire.

\section{RÉFÉRENCES}

I Dempsey JA, Reddan WG, Birmbaum ML, Foster HV, Thoden JS, Grover RF. et al. Effects of acute through life-long hypoxic exposure on exercise pulmonary gas exchange. Respir Physiol $1971 ; 3$ : 62-9.

2 Rasmussen J, Hanel B, Saunamaki K, Niels H, Secher NH. Recovery of pulmonary diffusing capacity after maximal exercise. J Sports Sci $1992 ; 10: 525-31$.

3 Caillaud C, Serre-Cousine O, Anselme F, Capdevilla X, Préfaut C. Computerized tomography and pulmonary diffusing capacity in highly trained athletes after performing a triathlon. J Appl Physiol $1995 ; 79: 1226-32$.

4 Hopkins SR, Schoene RB, Henderson WR, Spragg RG, Martin TR, West JB. Intense exercise impairs the ingrity of the pulmonary blood-gas barrier in elite athletes. Am J Resp Crit Care Med 1997; $155: 1090-4$.

5 Hopkins SR, Schoene RB, Henderson WR, Spragg RG, West JB. Sustained submaximal exercise does not alter the integrity of the lung blood-gas barrier in elite athletes. J Appl Physiol. 1998; 84 : $1185-9$.

6 Hanel B. Inge T, Alan R, Warberg J, Niels HS. Restricted postexercise pulmonary diffusion capacity and central blood volume depletion. J Appl Physiol $1997 ; 83: 11-7$. 